\title{
La motivación medida en sus factores extrínsecos e intrínsecos y su relación con la satisfacción laboral del personal del Colegio U nión, 2009
}

\author{
A rana Rodríguez, M aritza Soledad ${ }^{1}$ \\ 'Universidad Peruana Unión (UPeU), Perú
}

Recibido el 1 de abril de 2014 - A ceptado el 28 de octubre de 2014

\begin{abstract}
Resumen
A ctualmente son cada vez más las empresas que están considerando la satisfacción del personal como una ventaja competitiva que las diferencie de las demás. Por lo tanto, la medición de la Motivación y Satisfacción laboral constituyen una tarea necesaria como indicadores de la productividad y el desempeño laboral. El presente estudio tuvo como objetivo determinar la relación de la motivación medida en sus factores extrínsecos e intrínsecos y la satisfacción laboral del personal del Colegio Unión. El método fue descriptivo correlacional canónica y de corte transversal; la población estuvo conformada por 51 trabajadores del Colegio Unión; la técnica fue la encuesta y el instrumento una ficha de evaluación tipo cuestionario con escala de Likert, encontrándose los siguientes resultados: Existe relación conjunta significativa entre las variables predictoras factores extrínsecos 0 de higiene y factores intrínsecos 0 motivadores (Motivación) y las variables de criterio contenido del trabajo, trabajo en equipo, incentivos laborales, condiciones de trabajo (Satisfacción laboral), (Hotellings=1.872, $p=.000$ ). Los coeficientes de determinación múltiples son altamente significativos para las variables de criterio con relación al conjunto de variables predictoras (Contenido de trabajo $r_{1}=.282, p=.000$, Trabajo en equipo $r_{2}=.194$, $p=.006$, Incentivos laborales $r_{3}=.564, p=.000$, Condiciones de trabajo $\left.r_{4}=.399, p=.000\right)$. En conclusión la motivación presenta correlaciones altamente significativas con las variables de criterio: contenido de trabajo, trabajo en equipo, incentivos laborales, condiciones de trabajo y con la satisfacción laboral.
\end{abstract}

Palabras claves: motivación, satisfacción laboral, productividad y desempeño laboral.

\begin{abstract}
A bstract
Currently there are more and more companies that are considering personal satisfaction as a competitive advantage that differentiates them from the others. Therefore the measurement of motivation and job satisfaction is a necessary task as indicators of productivity and job performance. This study aimed to determine the relationship of the measured motivation in their extrinsic and intrinsic factors and job satisfaction of staff of the Union School. The method was descriptive, canonical correlation and cross sectional; population consisted of 51 workers of the Union School, the technique was the survey and the instrument an evaluation sheet type Likert scale questionnaire, following results were found: There is significant joint relationship between the predictor variables extrinsic or hygiene factors and intrinsic factors or motivators (motivation) and the criterion variable work content, teamwork, incentives, working conditions (job satisfaction), (Hotellings $=1.872, p=000$ ). The multiple determination coefficients are highly significant for criterion variables in relation to the set of predictor variables (job content $r_{1}=.282, p=.000$, Teamwork $r_{2}=.194, p=.006$, W ork Incentives $r_{3}=.564, p=$. 000 , Working conditions $\left.r_{4}=399, p=.000\right)$. In conclusion, the motivation presents highly significative correlations with criterion variables: work content, teamw ork, incentives, working conditions and job satisfaction.
\end{abstract}

Keywords: motivation, job satisfaction, productivity and job performance.

\section{Introducción}

En todos los ámbitos de la existencia humana interviene la motivación como mecanismo para lograr determinados objetivos y alcanzar determinadas metas, ya que representa un fenómeno humano universal de gran trascendencia para los individuos y la sociedad, es un tema de interés para quienes dirigen las organizaciones e instituciones en el ámbito mundial.

Esto debido a que, en los últimos años, las organizaciones han tenido una característica en común que ha consistido en comenzar a reconocer la trascendencia del potencial humano en el logro de los objetivos organizacionales, así como el ser competitivas en el entorno de su actividad.

Por esta razón se considera a la motivación de mucha importancia en cual quier ámbito o área; orientándonos al ámbito laboral se puede lograr que los empleados motivados se esfuercen por tener un mejor desempeño en su trabajo. A simismo, la evaluación de la satisfacción laboral de los empleados es útil para determinar si existen problemas en relación a los puestos o cargos designados al personal, ya que esta es producto del ambiente laboral que la dirección proporciona a través de sus directivas y políticas organizacionales.

Correspondencia al autor:

marana@upeu.edu.pe 
Sabemos que las organizaciones actuales están inmersas en cambios tecnológicos, sociales y económicos, y nadie duda que del proceso de adaptación a estos obtendremos los objetivos y metas propuestos, para lo cual es esencial disponer de personas motivadas y satisfechas con su trabajo y con la organización, esto aumentará claramente su rendimiento y la calidad del servicio que prestan.

En el campo de la educación; la motivación y la satisfacción laboral del personal son esencialmente relevantes en los profesionales tanto docentes como administrativos de un colegio, cuya razón de ser, es brindar un servicio educativo integral de calidad a quienes están a su cargo. Por esta razón el conocimiento y comprensión del nivel de motivación y de satisfacción del personal de un colegio constituyen información importante para la administración.

En este sentido, la investigación se propuso atender el problema ¿cuál es la relación de la motivación medida en sus factores extrínsecos e intrínsecos con la satisfacción laboral del personal del Colegio Unión? Con el propósito de atender el problema, el estudio se comprometía evaluar el grado de relación de dos conjuntos de variables: la motivación y la satisfacción laboral, partiendo de la hipótesis de que la motivación tiene una relación muy significativa con la satisfacción laboral, cuya organización muy sistémica ha permitido culminar con éxito la investigación.

El contenido de la investigación se ha organizado y estructurado atendiendo el protocolo establecido: la presentación de la problemática de estudio, los antecedentes, la justificación, la viabilidad y sus respectivos objetivos, presentados en el primer capítulo, luego se ha abordado sus fundamentos teóricos, transitando por el marco histórico y el marco teórico; en el primero se ha trabajado el marco histórico de la motivación y de la satisfacción laboral; por su parte en el marco teórico, se ha referido la motivación: las teorías, entendiendo que la motivación tiene dos grandes vertientes: la motivación extrínseca basada más en premios e incentivos que otorga el entorno para que las personas lleguen a ciertos resultados deseados y la motivación intrínseca manejada más por la propia persona que quiere conseguir por sí misma el desarrollo de su propio potencial y obtener el éxito en lo que hace; mientras que en la satisfacción laboral hemos suscrito los antecedentes de nuestra investigación, entendiendo que esta se mide a través del contenido del trabajo, trabajo en equipo, incentivos laborales y condiciones de trabajo; asimismo nos hemos ocupado sobre el marco conceptual, las variables y los indicadores de investigación, registrados en el segundo capítulo. La metodología de la investigación se ha tratado en el tercer capítulo, haciendo detalles específicos sobre el tipo de investigación, el diseño de la investigación, las delimitaciones, también la población y la muestra, asimismo, las técnicas y los instrumentos determinados para la recolección de datos, luego las técnicas y los análisis de los procedimientos de los datos. Finalmente, se ha abierto un espacio muy interesante para el cuarto capítulo, en el cual se ha abordado la información demográfica de los participantes, se ha realizado los análisis relacionados con las medias, así como el análisis de regresión multivariado de las dos variables: la motivación y la satisfacción laboral.

Llegando a la conclusión de que la motivación se relaciona significativamente con la satisfacción laboral, se podrán aplicar entonces medidas conducentes a incentivar la satisfacción del personal del Colegio U nión.

\section{Método}

A la luz de los estudios y contribuciones realizados por los investigadores presentados en el marco teórico, se observa que la motivación juega un papel preponderante en el comportamiento individual, además una capacidad predictiva más precisa es muy valiosa para mejorar la satisfacción laboral del trabajador.

\section{Hipótesis principal}

La motivación medida en sus factores extrínsecos e intrínsecos tiene una relación significativa con la satisfacción laboral del personal del Colegio Unión.

\section{Hipótesis específicas}

H1: Los factores extrínsecos o de higiene tienen una relación significativa con la satisfacción laboral medida del personal del Colegio Unión.

H2: Los factores intrínsecos o motivadores tienen una relación significativa con la satisfacción laboral del personal del Colegio U nión.

\section{Variablepredictora}

Motivación del trabajador medida en sus factores extrínsecos 0 de higiene y factores intrínsecos 0 motivadores.

\section{Variabledecriterio}

Satisfacción laboral con sus dimensiones: contenido del trabajado, trabajo en equipo, incentivos laborales y condiciones de trabajo. 


\section{O peracionalización de variables}

Tabla 1. Matriz instrumental

MEPIC - Matriz de la estructura del problema de investigación

METODO DELA CIENCIA Rodriguez.

2) INTERES DE LA INVESTIGACIÓN
(Realizar une breve descripción) Se quiere identificar cuales son las CAUSAS (relación o influencia) de la Insatisfacción laboral del personai del Colegio
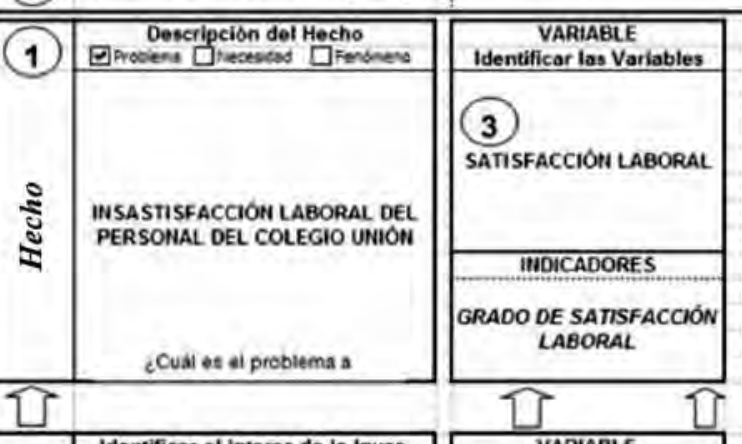

Unión
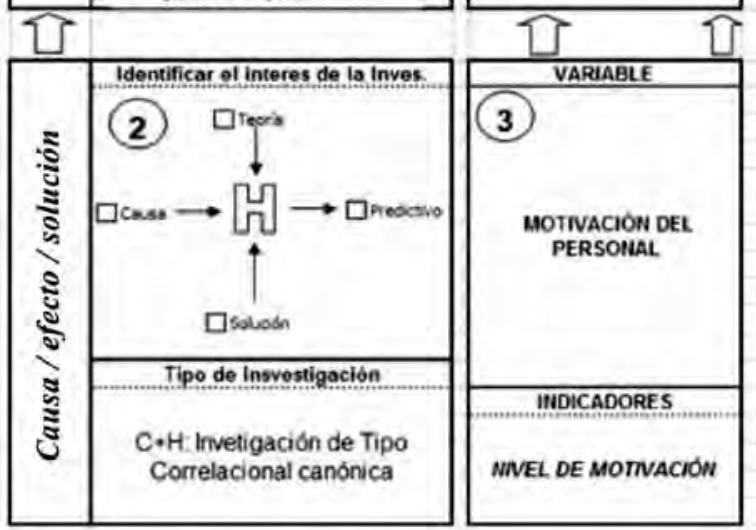

\begin{tabular}{|c|c|}
\hline \multicolumn{2}{|r|}{ PRE - OPERACIOHLALIZACIOH DE VARIABLES } \\
\hline DILENSION & ITEMS S/ SUB IIIDICADORES \\
\hline \multirow{4}{*}{ CONTENIDO DEL TRABAJO } & 1.1 Posicididad de Tomar decisiones en el puesto de trabajo \\
\hline & i. 2 Posibilidad de identificar su aporte en el resultado final \\
\hline & 13 Aceptación del puesto actual \\
\hline & I4 El puesto de trabajo permite el desarollo de sus habilidades \\
\hline \multirow[t]{2}{*}{ TRABAJO EN EOUIPO } & 2.1 El grado en que los miembros del gupo se sienten unidos \\
\hline & 22 El ambiente veinante dentro del grupo \\
\hline \multirow{4}{*}{ INCENTIVOS LABORALES } & 23 La comunicacion entre el personal \\
\hline & 3 i E nivel de correspondencia entre lo que el trabajador hace $y$ lo que recibe \\
\hline & 32 Efoctivoo derechos laborales \\
\hline & 33 Adecuados beneficios sociales \\
\hline \multirow[t]{2}{*}{ CONDICIONES DE TRABAJO } & 44 Horano de trabage \\
\hline & 45 Grado de aeguridad e higiene \\
\hline Identificarlas dimension*s & Identificar los indicadores por cada dimensión \\
\hline DIMENSION & TTEUS I SUB HIDICADORES \\
\hline \multirow[t]{7}{*}{ FACTORES EXTRINSECOS } & 11 Salaries justos \\
\hline & 12 Descansos vacacionales \\
\hline & 13 Beneficios sociales \\
\hline & II Rolaciones interpersonales positivas con sus comparteros \\
\hline & 15 Thato Justo \\
\hline & i 6 las politicas administrativas (normas) \\
\hline & If Condiciones generales de trabajo \\
\hline \multirow{9}{*}{ FACTORES IWTRINSECOS } & 21 E contenido del cargo \\
\hline & 22 La naturaleza de la tarea \\
\hline & 23 Elogio \\
\hline & 24 La responsabildad \\
\hline & 2.5 E reconocimiento \\
\hline & 2.6 La posibilidad de desarrollo \\
\hline & 27 El ascenso \\
\hline & 28 Deseo do auperacion constante \\
\hline & 29 Relaciones interperionales positivas con ous jetes \\
\hline
\end{tabular}

\section{Definción instrumental}

Cuestionario de evaluación de motivación y satisfacción laboral.

\section{Tipo de investigación}

La investigación es descriptiva, se aplica el modelo de correlación canónica, dado que, describe el comportamiento de la variable predictora motivación medida en sus factores extrínsecos e intrínsecos sobre la variable satisfacción laboral en sus dimensiones contenido del trabajo, trabajo en equipo, incentivos laborales y condiciones de trabajo y por qué mide la relación entre las variables que interactúan.

\section{Diseño de investigación}

El presente trabajo de investigación de acuerdo a Hernández (2003) es no experimental pues el investigador ni controla, ni manipula las variables de estudio sino que estas se dan independientes de la voluntad del investigador y de corte transversal pues se levantó información por única vez de la motivación y la satisfacción del personal y se analizó su relación.

Para probar la relación se utilizó el modelo estadístico de correlación.

$$
\begin{aligned}
& H_{0}: r=0 \quad \text { (Bilateral) } \\
& H_{1}: r \neq 0 \\
& \mathrm{M}(\mathrm{FE}, \mathrm{FI}) \rightarrow \mathrm{SL} \quad(\mathrm{CT}, \mathrm{TE}, \mathrm{IL}, \mathrm{CT})
\end{aligned}
$$

Dónde:

$M=$ M otivación al trabajador

$\mathrm{FE}=$ Dimensión factores extrínsecos 0 de higiene.

$\mathrm{FI}=$ Dimensión factores intrínsecos o motivadores.

$\mathrm{SL}=$ Satisfacción laboral

$\mathrm{CT}=$ Dimensión contenido del trabajo

$T E=$ Dimensión trabajo en equipo

$\mathrm{IL}=$ Dimensión Incentivos laborales

$\mathrm{CT}=$ Dimensión condiciones de trabajo

\section{Población y muestra}

En el presente trabajo de investigación, la unidad de observación quedó constituida por el número total del personal del Colegio Unión, el cual corresponde a 51 trabajadores.

Por el tamaño de la población, participaron la totalidad del personal, presentando un tipo de estudio censal. 


\section{Técnicas e instrumentos de recolección de datos}

\section{Técnicas de recolección de datos}

La técnica que se utilizó para levantar la información de los datos fue la encuesta y el instrumento fue el cuestionario (Hernández, 2003). Para iniciar la recolección de datos se solicitó la autorización (verbal) del área de estudio, mediante una entrevista con la directora del Colegio Unión a fin de obtener la autorización y facilidades para la realización del presente estudio. A sí como el permiso correspondiente de la administración central de la Universidad Peruana Unión. Se solicitó el apoyo a cada una de las personas que fueron encuestadas, explicando los objetivos del estudio a realizar y garantizándoles la confidencialidad de los datos.

El instrumento estuvo constituido por 41 ítems, distribuidos de la siguiente manera: 4 correspondían a la información general, 20 a la motivación y 17 a la satisfacción laboral del personal. La aplicación del cuestionario se dio en el tiempo de 20 minutos.

En la motivación se obtuvo la calificación máxima de 100 puntos, en sus dimensiones: factores extrínsecos 0 de higiene 45 puntos y en los factores intrínsecos 0 motivadores 55 puntos.

En la satisfacción laboral se obtuvo la calificación máxima de 85 puntos, considerando sus dimensiones: contenido de trabajo con 25 puntos, trabajo en equipo, incentivos laborales y condiciones de trabajo con 20 puntos respectivamente.

\section{A nálisis de los datos}

\section{Análisis de regresión multivariado - MANOVA entre: motivación y satisfacción laboral}

Considerando el análisis realizado entre motivación y satisfacción laboral, la tabla 6 , sugiere que existe relación conjunta significativa entre las variables predictoras factores extrínsecos 0 de higiene y factores intrínsecos o motivadores (M otivación) y las variables de criterio contenido del trabajo, trabajo en equipo, incentivos laborales, condiciones de trabajo (Satisfacción laboral), (Hotellings $=1.872, F(8,88)=$ $10.297, p=.000)$
Tabla 6. Análisis de regresión multivariada - M ANOVA

\begin{tabular}{llllll}
\hline Test & Valor & $\mathrm{F}$ & $\mathrm{GL}$ & Error GL & P_value \\
\hline Pillais & 0.807 & 7.772 & 8 & 92 & .000 \\
Hotellings & 1.872 & 10.297 & 8 & 88 & .000 \\
Wilks & 0.308 & 9.014 & 8 & 90 & .000 \\
Roys & 0.622 & & & & \\
\hline
\end{tabular}

A sí mismo corroborando nuestras hipótesis, la tabla 7 muestra los coeficientes de determinación múltiples altamente significativos para las variables de criterio: contenido del trabajo, trabajo en equipo, incentivos laborales, condiciones de trabajo (Satisfacción laboral) con relación al conjunto de variables predictoras: factores extrínsecos o de higiene y factores intrínsecos 0 motivadores (M otivación)

(Contenido de trabajo $r_{1}=.282, p=.000$, Trabajo en equipo $r_{2}=.194, p=.006$, Incentivos laborales $r_{3}=.564$, $p=.000$, Condiciones de trabajo $r_{4}=.399, p=.000$ ).

Es importante destacar que el $56.4 \%$ de la satisfacción originada por los incentivos laborales están siendo explicadas por la motivación tanto en sus factores extrínsecos e intrínsecos.

Tabla 7. Análisis M ANOVA entre factores extrínsecos 0 de higiene y factores intrínsecos o motivadores

\begin{tabular}{lllllll}
\hline & Sq. & Mul. & \multicolumn{5}{l}{ Hypoth. } \\
Variable & R & Adj. R-Sq. & MS & Error MS & F & P_Value \\
\hline Cont_tra & 0.282 & 0.253 & 94.639 & 10.016 & 9.449 & 0.000 \\
T_equipo & 0.194 & 0.161 & 27.866 & 4.814 & 5.788 & 0.006 \\
I_labora & 0.564 & 0.546 & 210.582 & 6.780 & 31.057 & 0.000 \\
Cond_tra & 0.399 & 0.374 & 135.841 & 8.510 & 15.963 & 0.000
\end{tabular}

El factor intrínseco influye significativamente en la satisfacción en el contenido de trabajo ( $B=0.332$, $p=.000$ ), los factores extrínsecos e intrínsecos influyen significativamente en la satisfacción de los incentivos laborales ( $B=0.352, \quad p=.000 ; \quad B=0.172, p=.020)$, así también los factores extrínsecos e intrínsecos influyen significativamente en la satisfacción de las condiciones de trabajo ( $B=0.203, p=.018 ; \quad B=0.221, p=.008$ ). Respecto a los factores motivadores que no influyen significativamente en la satisfacción laboral, se tiene al factor extrínseco en la satisfacción en el contenido del trabajo y tanto el factor extrínseco e intrínseco en el trabajo en equipo (ver Tabla 8). 
Tabla 8. Regresión múltiple multivariada entre las variables predictoras: factores extrínsecos 0 de higiene y factores intrínsecos o motivadores (Motivación) sobre las variables de criterio: Contenido del trabajo, trabajo en equipo, incentivos laborales, condiciones de trabajo (Satisfacción laboral).

\begin{tabular}{|c|c|c|c|c|c|c|c|}
\hline Covariable & B & Beta & Std. Err. & $\mathrm{T}$ & P_value & U. $.95 \%$ & LS. $95 \%$ \\
\hline F_extrin & .0 .047 & -0.078 & 0.09 & -0.522 & .604 & .0 .228 & 0.134 \\
\hline F_intrin & 0.332 & 0.572 & 0.087 & 3.835 & .000 & 0.158 & 0.506 \\
\hline \multicolumn{8}{|c|}{ Trabajo en equipo } \\
\hline Covariable & B & Beta & Std. Err. & $\mathrm{T}$ & P. value & U. $.95 \%$ & LS. $95 \%$ \\
\hline F_extrin & 0.076 & 0.193 & 0.062 & 1.22 & .228 & $\cdot 0.049$ & 0.202 \\
\hline F_intrin & 0.114 & 0.301 & 0.06 & 1.902 & .063 & .0 .007 & 0.235 \\
\hline \multicolumn{8}{|c|}{ Incentivos laborales } \\
\hline Covariable & B & Beta & Std. Err. & $\mathrm{T}$ & $P$ value & U.95\% & LS. $95 \%$ \\
\hline F_extrin & 0.352 & 0.554 & 0.074 & 4.761 & .000 & 0.204 & 0.501 \\
\hline$F_{\text {_intrin }}$ & 0.172 & 0.281 & 0.071 & 2.415 & .020 & 0.029 & 0.315 \\
\hline \multicolumn{8}{|c|}{ Condiciones de trabajo } \\
\hline Covariable & B & Beta & Std. Err. & $t$ & P_value & U. $95 \%$ & LS- $95 \%$ \\
\hline F_extrin & 0.203 & 0.334 & 0.083 & 2.446 & .018 & 0.036 & 0.37 \\
\hline$F$ intrin & 0.221 & 0.378 & 0.08 & 2.769 & .008 & 0.061 & 0.382 \\
\hline
\end{tabular}

Según el análisis de correlación (ver tabla 9), los factores motivadores extrínsecos presentan correlaciones altamente significativas con las variables de criterio trabajo en equipo, incentivos laborales, condiciones de trabajo y con la satisfacción laboral global $(r=.365, .715, .551, .799)$; los factores motivadores intrínsecos presentan correlaciones altamente significativas con todas las variables de criterio: contenido de trabajo, trabajo en equipo, incentivos laborales, condiciones de trabajo y con la satisfacción laboral global ( $r=.528, .412, .599, .570$, 841), la motivación global presenta correlaciones altamente significativas con todas las variables de criterio: contenido de trabajo, trabajo en equipo, incentivos laborales, condiciones de trabajo y con la satisfacción laboral global

$(r=.442, .438 . .739, .632, .925)$

Tabla 9. Correlaciones bivariadas de Pearson entre las variables predictoras y las de criterio

\begin{tabular}{|c|c|c|c|c|c|c|c|c|}
\hline & F_extrin. & F_entrin. & P motr. & Cont_tra. & T_equipo & I_laboral & Cond_tra & P_satist. \\
\hline F_extrin. & 1 & & & & & & & \\
\hline$F$ intrin. & $574^{\prime \prime}$ & 1 & & & & & & \\
\hline P_motiv. & $882^{\prime \prime}$ & $.892^{\prime \prime}$ & 1 & & & & & \\
\hline Cont_tra & .250 & .528 & $.442^{\prime \prime}$ & 1 & & & & \\
\hline T_equipo & $.365 "$ & $.412^{\prime \prime}$ & $.438^{\prime \prime}$ & $.485^{\prime \prime}$ & 1 & & & \\
\hline I_laboral & $.715 "$ & $.599^{-}$ & $.739^{\circ}$ & $.496^{\prime \prime}$ & $.318^{\circ}$ & 1 & & \\
\hline Cond_tra & $.551 "$ & $.570^{-1}$ & $.632^{-"}$ & $.661^{\prime \prime}$ & $.444^{-\prime}$ & $.692^{-\prime}$ & 1 & \\
\hline P_satist. & .799 & $.841^{-1}$ & $.925^{-1}$ & $.6899^{-1}$ & .582 & $.840^{-1}$ & $.824^{-}$ & 1 \\
\hline
\end{tabular}

\section{Interpretación de los resultados en relación a la hipótesis dela investigación}

La presente investigación nos exigió trabajar las hipótesis sobre la base del marco teórico:

HG: La M otivación medida en sus factores extrínsecos e intrínsecos tiene una relación significativa con la satisfacción laboral en sus dimensiones contenido del trabajo, trabajo en equipo, incentivos laborales y condiciones de trabajo del personal del Colegio Unión.

Como se puede apreciar en la tabla 6. El análisis de varianza multivariado (MANOVA), muestra que existe relación conjunta significativa entre las variables predictoras factores extrínsecos o de higiene y factores intrínsecos o motivadores (M otivación) y las variables de criterio contenido del trabajo, trabajo en equipo, incentivos laborales, condiciones de trabajo (Satisfacción laboral). (Hotellings=1.872, $F(8,88)=10.297, p=.000)$.

Además en la tabla 9 Correlaciones bivariadas de Pearson, encontramos también que la motivación global presenta una correlación altamente significativa con la satisfacción laboral global $(r=.925)$. En este sentido se acepta la hipótesis principal HG.

\section{Contrastación de las hipótesis espeáficas}

Sobre la relación de los factores extrínsecos o de higiene y de los factores intrínsecos o motivadores con la satisfacción laboral se planteó las siguientes hipótesis específicas:

H1: Los factores extrínsecos o de higiene tienen una relación significativa con la satisfacción laboral medida en sus dimensiones contenido del trabajo, trabajo en equipo, incentivos laborales y condiciones de trabajo del personal del Colegio Unión.

H2: Los factores intrínsecos o motivadores tienen una relación significativa con la satisfacción laboral medida en sus dimensiones contenido del trabajo, trabajo en equipo, incentivos laborales y condiciones de trabajo del personal del Colegio Unión.

Según los datos registrados en la tabla 7 A nálisis MANOVA entre variables de criterio y variables predictoras, los coeficientes de determinación múltiples son altamente significativos para las variables de criterio: contenido del trabajo, trabajo en equipo, incentivos laborales, condiciones de trabajo (Satisfacción laboral) con relación al conjunto de variables predictoras: factores extrínsecos 0 de higiene y factores intrínsecos 0 motivadores (Motivación). (Contenido de trabajo $r_{1}=.282, p=.000$, Trabajo en equipo $r_{2}=.194, p=.006$, 
Incentivos laborales $r_{3}=.564, p=.000$, Condiciones de trabajo $r_{4}=.399, p=.000$ ).

A demás en la tabla 9 el análisis de correlación muestra que los factores motivadores extrínsecos presentan correlaciones altamente significativas con las variables de criterio satisfacción en el trabajo en equipo, los incentivos laborales, las condiciones de trabajo y la satisfacción laboral global ( $r=.365 .715, .551, .799)$; los factores motivadores intrínsecos y la motivación global presentan correlaciones altamente significativas con todas las variables de criterio: satisfacción en el contenido de trabajo, el trabajo en equipo, los incentivos laborales, las condiciones de trabajo y la satisfacción laboral global ( $r=.442, .438 . .739, .632, .925)$. En este sentido se aceptan las hipótesis específicas $\mathrm{H} 1$ y H2.

\section{Condusión}

EI análisis de regresión multivariado (MANOVA), muestra que existe relación conjunta significativa entre las variables predictoras factores extrínsecos o de higiene y factores intrínsecos o motivadores (M otivación) y las variables de criterio contenido del trabajo, trabajo en equipo, incentivos laborales, condiciones de trabajo (Satisfacción laboral). Es decir la motivación, de una u otra manera, ha repercutido en el contenido del trabajo, el trabajo de equipo, incentivos laborales y condiciones de trabajo; evidenciándose de esta manera la demostración de la hipótesis principal. Por lo tanto, se puede predecir que la motivación puede lograr que los empleados motivados se esfuercen por tener un mejor desempeño en su trabajo. A simismo, la satisfacción laboral es producto del ambiente laboral, por tanto, la motivación y la satisfacción laboral del personal son esencialmente relevantes en los profesionales tanto docentes como administrativos de una institución educativa, cuya razón de ser es el poder brindar un servicio educativo integral de calidad a quienes estén a su cargo. Por esta razón el conocimiento y comprensión del nivel de motivación y de satisfacción del personal de un colegio constituye información importante para la administración.

\section{Referendias}

A liaga, Cecilia. (1999). Comportamiento Organizacional. Lima. Instituto de Investigación de la Facultad de ciencias A dministrativas de la universidad San M artin de Porres. Pág. 61.

A rana, Walter (2005). La motivación y la gestión de la dirección.

Recuperado el 12 de agosto del 2008, en la dirección electrónica:

http://www.gestiopolis.com/canales/motigestion.htm.

A rdilla, Rubén. (1986). Psicología del Trabajo. Chile. Editorial Universitaria. Pág. 46

B ernal, C. y Sierra, H. (2008). Proceso Administrativo para las organizaciones del Siglo XXI. M éxico. Pearson Educación S.A. Pág. 156

Chiavenato, I dal berto. (2004). Comportamiento Organizacional- $L$ a dinámica del éxito en las organizaciones. M éxico. International Thomson Editores. Pág. 313.

Chiavenato, I dal berto. (2009). Gestión del Talento H umano. M éxico. M c. G raw-Hill. Pág. 233

D aft, R. y Steers, R. (1992). Organizaciones. M éxico. Limusa. Pág. 136.
Davis, K eith. \& N ewstrom, John. (2003). Comportamiento H umano en el trabajo. M éxico. M c Graw-Hill. Pág. 172.

Dessler, Gary. (1979). Administración de Personal. M éxico. M c Graw-Hill. Pág. 121.

Dolan, S., V alle, R., Jackson, S. \& Schuler, R. (2007). La $\mathrm{G}$ estión de los Recursos humanos. M éxico. M c GrawHill. Pág.351.

Donnelly, Gibson e Ivancevich. (1994). F undamentos de la dirección y administración de empresas. España. Mc Graw-Hill. Pág. 303.

Dowlinng, W . F. \& Sayles, L.R. (1979). M ando y M otivación Efectiva del Personal. Barcelona. Editorial Hispano Europea. Págs. 6 y 36.

Dubin, Roberto. (1974). Relaciones humanas en la administración. M éxico. Cía. Editorial Continental S.A. Pág. 114.

Dubrin, A ndrew J . (2008). Relaciones humanasComportamiento humano en el trabajo. M éxico. Pearson Educación S.A. Pág.177.

García, Pacas \& Ramírez. Estudio Expost facto sobre la motivación y la satisfacción laboral. Recuperado el 18 
electrónica:http://www.monografias.com/trabajos36/sati sfaccionlaboral/satisfaccion-laboral.shtml.

Garza, J uan G. (2000). Administración Contemporánea. M éxico. M c Graw-Hill. Pág. 204.

G reat Place to W ork Institute Perú. Las 25 mejores empresas para trabajar en el Perú. Diario EI Comercio, Suplemento especial del 29 de noviembre del 2006.

Guillen, M anuel. (2006). Ética en la organizaciónConstruyendo confianza. M adrid. Pearson Edición S.A. Pág. 158.

Hackman \& Oldman. (1975). M otivación, satisfacción y desempeño. R ecuperado el 12 de setiembre del 2009, en la dirección electrónica: http://www.scribd.com/doc/21417589/

Hellriegel \& Scolum (2004). Comportamiento Organizacional. M éxico. International Thomson Editores S.A. Págs. 51,116 y 154.

Hellriegel, Scolum \& W oodman (1998). Comportamiento Organizacional. M éxico. International Thomson Editores. Pág. 136.

Hernández, S. R oberto (2003). M etodología de la Investigación. M éxico. M c G raw-Hill. Págs. 193,346 y 368.

Herzberg. La M otivación y la gestión de dirección. Recuperado el 15 de febrero del 2008, en la dirección electrónica: www.wikilearning.com/...teoria de los dos factore S.../16111-7

Hodgetts \& Altman. Motivación y satisfacción. Recuperado el 11 de agosto del 2009 en la dirección electrónica: http://www.educa.madrid.org/web/les/...madrid/.../M OTI VATION.doc

Hodgetts, Richard. \& A Itman, Steven. (1985). Comportamiento en las organizaciones. M éxico. Nueva editorial Interamericana S.A . Pág. 95.

Hoppock. M otivación, satisfacción y desempeño. Recuperado el 15 de setiembre del 2008, en la dirección electrónica: www.scribd.com/doc/.../M otivación-satisfacción-ydesempeño

K ast, Fremont \& R osenzweig, J ames. (1988). Administración en las 0 rganizaciones- Enfoque de sistemas y de contingencias. M éxico. M c G raw-Hill. Pág. 297.
Interamericana de M éxico S.A. M c Graw-Hill. Págs. 466 y 469 .

K oontz, H arold \& W eihrich, Heinz. (1998). Administración. M éxico. M c Graw-Hill. Págs. 508 y 517.

K reitner, R. \& Kinicki, A . (1999). Comportamiento de las Organizaciones. M éxico. M c Graw-Hill. Pág. 152

León F. \& Pejerrey G. ( 1978). Citado en Diagnóstico de Satisfacción Laboral en una empresa textil peruana. Recuperado el 05 de agosto del 2008. En http://monografias.com/trabajos13/

Locke. Estudio de Satisfacción Laboral. Recuperado el 21 de octubre del 2008, en la dirección electrónica: http://www.cybertesis.ubiobio.cl/tesis/2007/contreras_g/d oc/contrer as_g.pdf

M eliá, J.L. \& Peiró, J.M. Cuestionario de Satisfacción Laboral S4/82. Recuperado el 18 de febrero del 2008, en la dirección electrónica:

http://www.uv.es/meliaj//Research/Cuest_Satisf//S04_82. PDF

M ink, G. (2000). H ostile Enviroronment. Ithaca, NY . Oxford University. Pág. 163.

M uñoz (1989). Satisfacción e insatisfacción en el trabajo. Universidad Complutense de M adrid. Recuperado el 14 de agosto del 2008, en la dirección electrónica: http://www.cibernetia.com/tesis_es/psicologia_industrial /motivacion_laboral_y_actitudes.

Nelson, B ob. (2001) F ormas de M otivar. Recuperado el 09 de noviembre de 2008 en la dirección electrónica: http://www.nelsonmotivation.com. Pág. 3.

Palma S. (1999). Elaboración y Validación de una Escala de Satisfacción Laboral SL-SPC para trabajadores de Lima M etropolitana. Recuperado el 10 de junio del 2008 en la dirección electrónica: http://www.urp.edu.pe/urp//.../satisfaccion_laboral.php pág. 2

Palma, S. (2001). F actores para evaluar la Satisfacción Laboral. Recuperado el 6 de mayo del 2008 en la dirección electrónica: http://www.ucvvirtual.edu.pe/portal/escuelas/psicologia/ tesis ps/.../ T180.doc

Porret, M iguel. (2007). Recursos H umanos- Dirigir y gestionar personas en la organización. España. ESIC Editorial Libros Profesionales de empresa. Págs. 86 y 90. 
Revista de Investigación de la Fac. de Ciencias Administrativas. 2005. "Gestión en el Tercer Milenio".Vol. 8 № 15, Lima. UNM SM.

Robbins, Stephen. (2004). Comportamiento Organizacional . M éxico. Pearson Prentice Hall. Págs. 78 y 155.

Rodríguez, J oaquín. (2006). Dirección M oderna de Organizaciones. M éxico. International Thomson Editores. Pág.159.

Shouksmith, Pajo y J epsen. Escala M ultidimensional de Satisfacción en el Trabajo. Recuperado el 22 de febrero del 2008, en la dirección electrónica: http://www.cybertesis.ubiobio.cl/tesis/2007/

Smith, Kendall \& Hulin. M otivación, satisfacción y desempeño. Recuperado el 10 de setiembre del 2008, en la dirección electrónica: http://www. scribd.com $>$ B ooks $>$ N on-fiction

Soto, Eduardo. (2001). Comportamiento Organizacional. M éxico. Thomson Editores S.A. Pág. 107.

Stoner, J .,Freeman, E. \& Gilbert, D. (1996). Administración. M éxico. Prentice Hall Hispanoamericana. S.A. Pág. 484.

Tejada, K. (2007). Tesis de M aestría en A dministración sustentada el 17 de mayo de 2007, en la U niversidad de M ontemorelos , Facultad de Ciencias E mpresariales, titulada: "F actores predictores de la satisfacción laboral en los docentes de la U niversidad Adventista Dominicana y del Colegio Adventista Dominicano en el
2006" Recuperado el 10 de junio del 2008 en la dirección electrónica:

http://dspace.biblioteca.um.edu.mx/xmlui/handle/

V aldez, Enrique. (1998). Administración de Recursos Humanos. Lima. Escuela Superior de N egocios ESA N. Pág. 18.

V alencia, Pedro. (2008). Tesis de M aestría en A dministración, en la Universidad $M$ ayor de San M arcos. U nidad de Postgrado, titulada: "Estilos G erenciales y Satisfacción Laboral del personal administrativo de la Dirección General de Administración de U.N.M S.M." Recuperado el 18 de setiembre del 2009 en la dirección electrónica: http:

www.cybertesis.edu.pe/.../rtermes.xsp?...Estilos+gerencia les...satisfacción+laboral. Pág. 15

V elásquez (1982). Escala de Satisfacción Laboral. Recuperado el 23 de julio del 2008, en http://www.scribd.com/.../escala de satisfacción laboral.

V romm, V. \& Deci, E. (1979) La M otivación y alta dirección. M éxico. M c Graw Hill. Pág. 251.

Warr, Cook \& Wall. Satisfacción laboral: escala general de satisfacción. Recuperado el 17 de octubre del 2008, en la dirección electrónica:

http://www.mtas.es/insht/ntp/ntp_394.htm

W est, M ichael. (2004). Los Secretos de la G estión de los Equipos de Trabajo. Barcelona. B LU M E Empresa. Pág. 20. 